\title{
Study of Current Status and Development of Photoelectric Current Transformer Technology
}

\author{
Sun Haijiang, Hou Jibiao \\ Research institute of national grid smart grid \\ 16295036@qq.com
}

Keywords: Optical current transformer, technical difficulties, development, principle

\begin{abstract}
Aiming at photoelectric current transformer technology, this paper analyses the principle of passive and active transformer firstly, then discusses the main technical difficulties in this area currently and the development of optical current transformer. We hope all above can play a reference role for related work. With the gradually deepen theory research on photoelectric current transformer technology in domestic and the continuous improving achievements acquired in practice, it's necessary to study the current status and development of photoelectric current transformer technology.
\end{abstract}

\section{The principle of passive photoelectric current transformer}

\section{Passive type}

This type of transformer can be divided into two kinds based on the principle of the device of high pressure part of the sensor.One type is active,the other is passive.Compared with the active type,the sensing head of passive type doesn't need power supply.In general,it's designed based on the principle of Faraday magneto-optic effect . if the magnetic field direction keep in parallel with the direction of propagation of the linear polarized beam that is in the Faraday rotatory material,then the radiation polarization plane of the light will rotate.That is to say the magnetic field generated by current signal modulates polarization light-wave.

\section{Faraday magneto-optical effect}

The Faraday magneto-optic effect mentioned above refers to the rotation of light polarization plane that transmit light caused by the affect of applied magnetic field.In a magnetic field environment, if the direction of the magnetic field keep in parallel with the direction of propagation of the linear polarized beam that is in Faraday rotatory material, then the exit linear polarized light might share the same rotation angle with polarization plane of incident ray $\theta$.And the $\theta$ satisfied the formula

$$
\theta=\mu U d H L
$$

with:

$\mu$ _ Transmission of Faraday rotatory material

Ud_-Foer's constant( ras/A)

$\mathrm{H}-$ Magnetic field intensity $(\mathrm{A} / \mathrm{m})$

L__Length of optical path in Faraday rotatory material (m)

When the wavelength is in a certain case,and Faraday devices material in a certain range,then Ud is decided.For a certain material,parameters $\mu$ and $\mathrm{L}$ is a constant value.Obviously,rotation angel $\mu$ is proportional to magnetic field intensity.

\section{Operating principle}

The light goes through optical fiber reaches polarizer, where it is converted to the linear polarized light.Then by the impact of Faraday rotatory device, the polarization plane of the light will rotate a certain angel ,defined as $\theta$.Finally,measure $\theta$ by analyzer and transfer the optical signal into electrical signal by photoelectric detector. At the same time,we can reach the size of the magnetic field current (I). 


\section{The principle of active photoelectric current transformer}

\section{Active type}

Active photoelectric current transformer measure current by high voltage.Electrical signal can be delivered light-emitting component by sampling coil,then it is converted into optical signal.Now,signal can be passed by light to the low voltage side,where it inverse changes into external electrical signal that can be output.There three way to provide power to the high side device.

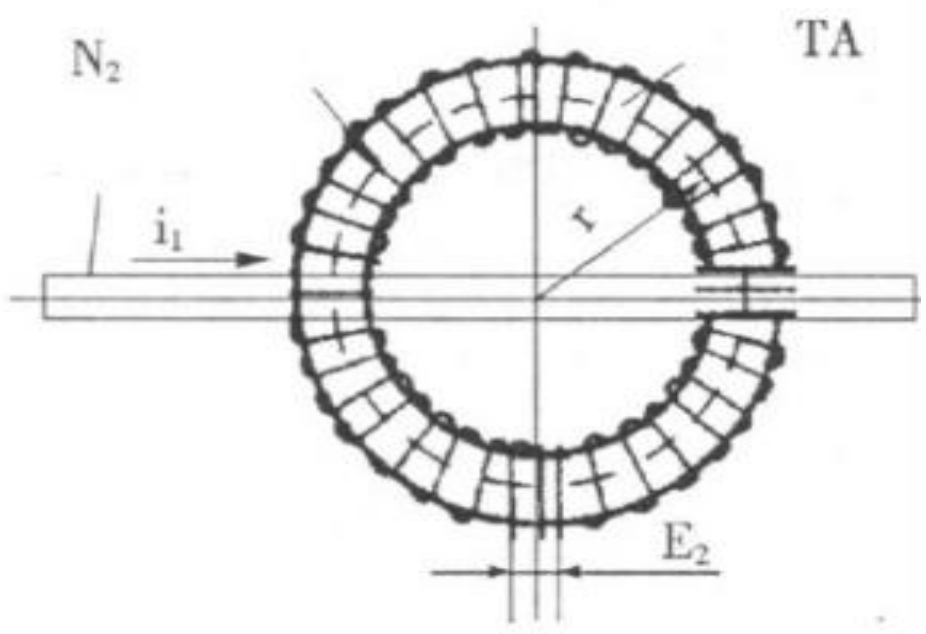

Figure 1 the structure of air-core coil

\section{Principle of Rogowski coil}

As a relatively mature measuring element,it's frequency response is good, accuracy is high and the structure is very simple,what's more,the cost of it is relatively low, therefore it's regarded as an ideal busbar current sampling device at present.Normally,the coil is like a air-core coil where wire is winded to non-magnetic skeleton regularly. As it would not contact with the circuit to be measured directly,Heat insulation measurement on high voltage circuit is simplified greatly. Thus it can be used in active electric current transformer.Figure 1 shows the structure of air-core coil.The coil is a non-magnetic ring skeleton winded by wire.In the picture,r means radius, $\mathrm{s}$ is cross area and

$\mathrm{E} 2$ is the secondary output voltage.As the Figure 1 shows, one wire through the hollow winding.As the skeleton is non-magnetic material,mutual inductance $M$ value in equivalent circuit is affected and the reactive resistance of load will be too high because of the low magnetic permeability.In practice application,its secondary voltage output is proportional to reciprocal value of the first current,thus the two parameters on the phase difference is ninety degrees.To avoid the difference,an integral part like RC is added into the circle.By the way the coil is put in screening can to avoid the interference of magnetic field around.

\section{Operating principle}

The principle of active photoelectric current transformer based on coil sampling is simple.While the high voltage side circuit is consisted of magnetic potentiometer, integral circuit and phase-shift circuit and so on.Rate of change of Induced voltage in Rogowski coil and the current to be measured are direct proportional.On the one hand,the output of integral and phase-shift circuit is proportional to the measured current I in phase.On the other hand,mold/data conversion electric current then converts analog signal from phase-shift circuit into data signals,thus the light source is driven,that means digital signal will be converted into optical signals again.By this way,the current to be detected can arrive in low voltage side signal processing circuit by optical fiber in the form of light digital signal.At the same time,the high side power guarantees actives devices run normally. 


\section{The main technical difficulties of photoelectric transformer study}

\section{The magnetic field}

Passive photoelectric transformer is based on the principle of magneto-optic effect,which also lead to measuring interference by the magnetic field around, so that the coming of measurement deviation.

\section{The power supply in high voltage side}

There is only one kind electromagnetic connection called conduction fiber between the high and low voltage side of active photoelectric transformer,naturally,power supply mode of high voltage side circuit has become one of the main technical researches at present.

\section{Specified integrator}

The output of Rogowski coil is current signal,while the later electronic circuit can only do with voltage signal.Thus a specified integrator is deadly needed in this transformer to convert current signal to voltage signal.Unluckily,the design of that integrator is difficult.

\section{The birefringence effect}

Nearly all the single mode fiber feature linear birefringence,caused by not completely symmetry in refractive index or diameter circular of fiber core structure.As it will affect the accuracy of measured values more or less,study on the birefringence effect and find out the corresponding solution becomes especially important on improving the accuracy of measurements and obtaining a more sensitive mutual inductor.

\section{Temperature}

The temperature of environment affect the Verdet constant of fiber material a lot.And the interrupted system result in deviation of measuring result.

\section{Vibration interference}

In the process of measurement,when optical system is disturbed,measuring result's error comes.But some unavoidable factors such as switch of circuit,surrounding environment as well as human disturbance are of vital importance.

\section{The development of the photoelectric current transformer}

This type of transformer is a collection of theory and application from many subjects,for example,optical fiber sensing technology and nonlinear optical and photoelectric technology are all adopted.In the early 1960s many developed countries have studied this subject.In 1963, the way by the device installed on $230 \mathrm{kv}$,employed glass waveguide to transmit signal successfully, which is the original form of this new type of interaction.in 1977, the research center in UK starting from the principle do constantly in-depth study on full optical fiber current sensor based on the principle and produced experimental device in lab successfully.By 1979 the pilot run in generating station made a success.Around 1982 the top five power companies set up OCT study group respectively,which later successfully developed $161 \mathrm{kv}$ detached OCT.By the middle of 1991,the T\&D co., LTD., of SBB released a OCT system with $345 \mathrm{kv}$ power station which can realize measurement and relay protection. After four months of operation test,they announce that its difference between standard current transformer is only $0.4 \%$.Then with a few years after that,voltage class of ac digital OTC is improved from $72.5 \mathrm{kv}$ to $800 \mathrm{kv}$ successfully.Meanwhile once-through OCT developed a lot too.Many regions are also admitted to run it.Domestic research in this field can trace back to 1980. Theoretical research and practice work drove by colleges and universities lay a foundation for its development and progress.As its theories grew mature and market demand for the expansion, some powerful groups and manufacturers ask to cooperate with the college.By the 1990s, Chinese electric power research and Tsinghua University developed the 110 kVOCT jointly and pilot run for test was conducted as well.Within a few years after that,a transformer substation in Guangdong lead a formal pilot run. Although the domestic researches on this aspect are more and more deep, there is still a gap compared with developed countries since the commercialization of the product is not realized by now. 


\section{Conclusion}

Photoelectric current transformer technology is an integration of theories and application from many subjects,the development disparity between domestic and foreign developed countries is still exist,some technological difficulties are faced in the research process. Therefore, continuously strengthened study on it is necessary so that we can meet the demand of society.

\section{References}

[1] Fan Ming,Zheng Jiana.For the Application of Photoelectric Current Transformer in High Voltage Watt-hour Meter J.Science and Technology Innovation and Application,2014,28:161.

[2] Chen Gang,Zhao Shuangshuang,Chen Mingming.Application of Optical Current Transformer Technology in Power System J.Electrical Application,2014,20:86-89. 